\title{
Structural Phase Transition in Liquid Crystal Doped with Gold Nanoparticles
}

\author{
P. KopČAnskÝ ${ }^{a}$, N. TOMAŠOViČOVÁ ${ }^{a}$, M. KonerackÁ ${ }^{a}$, M. TimkO $^{a}$, Z. Mitróová $^{a}$, \\ V. ZÁViŠOVÁ ${ }^{a}$, N. ÉBer ${ }^{b}$, K. Fodor-CsOrba ${ }^{b}$, T. TÓth-KATONA ${ }^{b}$, A. VAJdA $^{b}$, \\ J. JADZYN ${ }^{c}$, E. BEAUGNON ${ }^{d}$ AND X. CHAUD ${ }^{d}$ \\ ${ }^{a}$ Institute of Experimental Physics, Slovak Academy of Science, Watsonova 47, 04001 Košice, Slovakia \\ ${ }^{b}$ Research Institute for Solid State Physics and Optics, Hungarian Academy of Sciences
} H-1525 Budapest, P.O. Box 49, Hungary

${ }^{c}$ Institute of Molecular Physics, Polish Academy of Sciences, M. Smoluchowskiego 17, 60-179 Poznań, Poland

${ }^{d}$ High Magnetic Field Laboratory, CNRS, 25 Av. des Martyrs, Grenoble, France

The influence of the inclusion of the dodecanethiol functionalized gold particles (with diameter $3-5 \mathrm{~nm}$ ) on the structural transitions was investigated. The studied samples were based on the nematic liquid crystal 4 -(trans- $4^{\prime}-n$-hexylcyclohexyl)-isothiocyanatobenzene (6CHBT). The volume concentration of the gold particles was $\phi_{1}=2 \times 10^{-4}$ and $\phi_{2}=10^{-3}$. The obtained results showed that the inclusion of the gold particles in the 6 CHBT liquid crystal increases the sensitivity of such system on the external magnetic field.

PACS numbers: 75.50.Mm, 61.30.Gd, 61.30.Hn

\section{Introduction}

System of nanoparticles dispersed in liquid crystals have attracted attention for development of novel materials based on the controlled assembly of the particles. The properties of such materials, in most cases, significantly differ from those of the host materials. In the past few years there has been a significant increase in the number of published articles dealing with use of metallic, semiconducting, ferromagnetic or ferroelectric nanoparticles, fullerenes and carbon nanotubes in mixture with liquid crystals [1-4]. Depending on experimental parameters such as nanoparticle functionalization and concentration, size and core material, cell surface treatment, as well as applied electric or magnetic field diverse effects arise in different nematic liquid crystal hosts $[4,5]$. This effect is due to the anchoring of the liquid crystal molecules at the surface of the particles. The aim of the presented work was to study influence of the gold particle admixture on structural phase transition in nematic liquid crystal in electric and magnetic fields.

\section{Experiment}

The studied ferronematic samples were based on the thermotropic nematic 4-(trans-4'-n-hexylcyclohexyl)-isothiocyanatobenzene (6CHBT) [6]. The nematic sample was doped with the dodecanethiol functionalized gold nanoparticles of size 3-5 nm dispersed in toluene (concentration $2 \%$ ) purchased from Sigma-Aldrich Co. The volume concentration was $\phi_{1}=2 \times 10^{-4}$ and $\phi_{2}=10^{-3}$, respectively. The doping was done by adding this suspension, under continuous stirring, to the liquid crystal in the isotropic phase. This admixture shifts the temperature of the nematic-isotropic transition to lower values depending on the volume concentration of admixture, so the measurements in the magnetic and electric fields were done at $25^{\circ} \mathrm{C}$, i.e. at temperature when all samples (undoped and doped) are in nematic state.

The structural transitions were monitored by capacitance measurements in a capacitor made of ITO-coated glass electrodes (LINCAM Co.). The capacitor with the electrode area approximately $1 \mathrm{~cm} \times 1 \mathrm{~cm}$ was connected to a regulated thermostat system, the temperature was stabilized with the accuracy of $0.05^{\circ} \mathrm{C}$. The distance between the electrodes (sample thickness) was $D=5 \mu \mathrm{m}$. The capacitance was measured at the frequency of $1 \mathrm{kHz}$ by the high precision capacitance bridge Andeen Hagerling.

\section{Results and discussion}

The structural transitions in combined electric and magnetic fields were studied in the experimental geometry shown in Fig. 1. We start from a planar aligned cell. During measurements the external magnetic field was applied parallel to the capacitor electrodes and the electric field was applied perpendicular to the capacitor electrodes. First, the initial planar alignment was stabilized with the external magnetic field and then the electric field was increasing until the director of sample was reoriented to the electric field direction. The distortion manifests itself in the increase of the capacitance that reflects the re-orientation of the nematic molecules. Figures 2 and 3 show the dependence of the capacitance on the electric field at different magnetic fields of $6 \mathrm{CHBT}$ liquid crystal doped with volume concentration of gold particles $\phi_{1}$ and $\phi_{2}$, respectively. These dependences show that the critical electric field is shifted to higher values with the increase of the magnetic field.

The dependences of the critical electric fields on the applied magnetic field for pure 6CHBT and 6CHBT doped with different volume concentration of the gold nanoparticles are shown in Fig. 4. In the given geometry the electric threshold $U$ in the presence of a magnetic field 

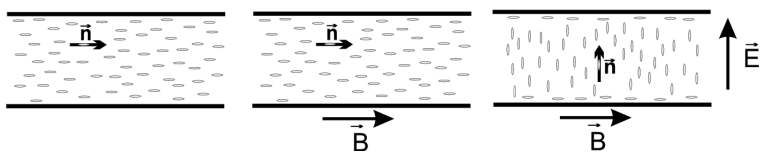

Fig. 1. Cross-section of the cell in the initial state, after application of the magnetic field and after application of the voltage $U>U_{\mathrm{c}}$. The unit vector $\boldsymbol{n}$ (director) denotes the preferential direction of the nematic molecules.

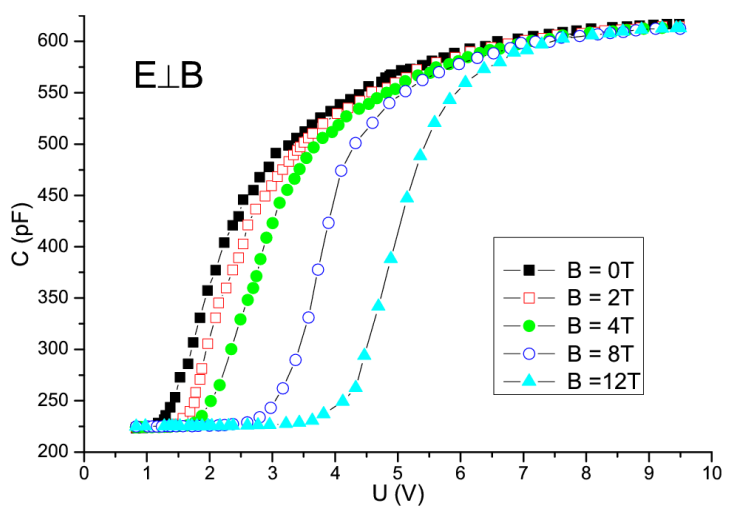

Fig. 2. Capacitance dependence of 6 CHBT doped with gold particles with volume concentration $\phi_{1}=2 \times 10^{-4}$ on electric field measured at different bias magnetic fields.

$H$ is given by the formula

$$
\left(\frac{U}{U_{\mathrm{F}}}\right)^{2}=1+\left(\frac{H}{H_{\mathrm{F}}}\right)^{2}
$$

where $U_{\mathrm{F}}$ is the critical voltage of the electric Freedericksz transition at $H=0$ and $H_{\mathrm{F}}$ is the magnetic Freedericksz threshold field (at $U=0$ ). In the experiment the threshold of the structural transition of the pure liquid crystal and the liquid crystal doped with different volume concentration of gold particles were defined as the voltage at which the capacitance variation (indicating the distortion of the director) reached $10 \%$ of its maximum.

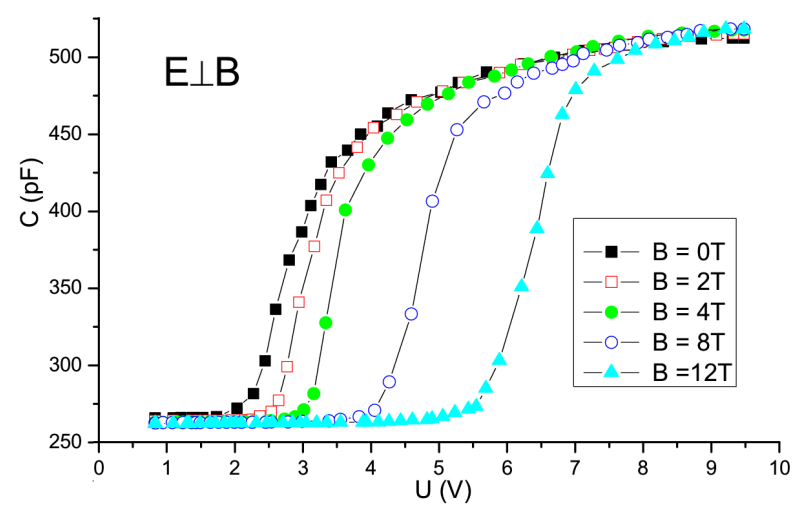

Fig. 3. Capacitance dependence of $6 \mathrm{CHBT}$ doped with gold particles with volume concentration $\phi_{2}=10^{-3}$ on electric field measured at different bias magnetic fields.

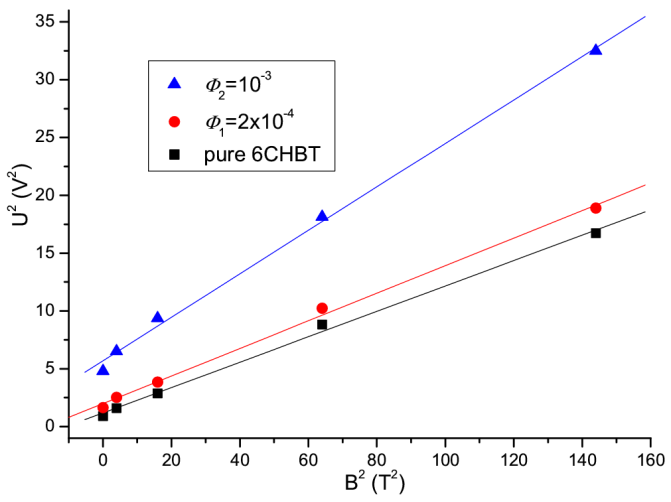

Fig. 4. Dependence of the critical electric fields on the bias magnetic field for pure 6CHBT and 6CHBT doped with gold particles with volume concentration $\phi_{2}=2 \times 10^{-4}$ and $\phi_{2}=10^{-3}$. The solid lines represent the best linear fit to Eq. (1).

\section{Conclusion}

The influence of the inclusion of the dodecanethiol functionalized gold particles (with diameter $3-5 \mathrm{~nm}$ ) to 6CHBT liquid crystal on the structural transitions was investigated. The obtained results showed that doping with dodecanethiol functionalized gold particles changes the sensitivity of the nematic liquid crystal $6 \mathrm{CHBT}$ on the external magnetic field.

\section{Acknowledgments}

This work was supported by the Slovak Academy of Sciences grant VEGA 0077 and Center of Excellence Nanofluid, APVV-0509-07 and SK-HU-0008-08, Hungarian Research Funds OTKA K61075 and NKTH-TÉT SK-12/2008, the Grenoble High Magnetic Field Laboratory, with support of EC from the 7th FP capacities "Transnational Access" - Contract No. 228043 - EuroMagNET II, and with support by European Regional Development Fund project 26220120021.

\section{References}

[1] F. Haraguchi, K. Inoue, N. Toshima, S. Kobayashi, K. Takatoh, Jpn. J. Appl. Phys. 46, 796 (2007).

[2] V.Y. Reshetnyak, S.M. Shelestiuk, T.J. Sluckin, Mol. Cryst. Liq. Cryst. 454, 201 (2006).

[3] L.M. Lopatina, J.V. Selinger, Phys. Rev. Lett. 102, 197802 (2009).

[4] P. Kopčansky, N. Tomašovičová, M. Koneracká, V. Závišová, M. Timko, A. Džarová, A. Šprincová, N. Éber, K. Fodor-Csorba, T. Tóth-Katona, A. Vajda, J. Jadzyn, Phys. Rev. E 78, 011702 (2008).

[5] L.J. Martínez-Miranda, L.K. Kurihara, J. Appl. Phys. 105, 084305 (2009).

[6] R. Dabrowski, J. Dziaduszek, T. Szczucinski, Mol. Cryst. Liq. Cryst. Lett. 102, 155 (1984). 